\title{
Extension of the Rocchio Classification Method to Multi-modal Categorization of Documents in Social Media
}

\author{
Amin Mantrach and Jean-Michel Renders \\ Yahoo! Research Barcelona ${ }^{\star}$ Xerox Research Centre Europe \\ amantrac@yahoo-inc.com, jean-michel.renders@xrce.xerox.com
}

\begin{abstract}
Most of the approaches in multi-view categorization use early fusion, late fusion or co-training strategies. We propose here a novel classification method that is able to efficiently capture the interactions across the different modes. This method is a multi-modal extension of the Rocchio classification algorithm - very popular in the Information Retrieval community. The extension consists of simultaneously maintaining different "centroid" representations for each class, in particular "crossmedia" centroids that correspond to pairs of modes. To classify new data points, different scores are derived from similarity measures between the new data point and these different centroids; a global classification score is finally obtained by suitably aggregating the individual scores. This method outperforms the multi-view logistic regression approach (using either the early fusion or the late fusion strategies) on a social media corpus - namely the ENRON email collection - on two very different categorization tasks (folder classification and recipient prediction).
\end{abstract}

\section{Introduction}

Multi-modal (or multi-view1 ${ }^{1}$ ) learning has been intensively studied since a long period. It relates to the problem of learning from multiple set of features. As suggested by [1], the multi-modal learning takes its justification from the fact that a high consensus of two independent hypotheses results in a low generalization error. These last years, several new methods have been proposed for the semi-supervised and the unsupervised settings (for semi-supervised multiview learning, see the survey in [2]). However, few has been done in the fully supervised setting.

Actually, as pointed out by [3], in a fully supervised setting, multi-modal learning usually performs worse than learning on the union of all modes. In this setting, the standard approaches are the early fusion $(E F)$ method and the late fusion $(L F)$ method. The former consists of directly learning from a common

* This work has been done when the author was with Xerox Research Centre Europe.

1 The multi-view learning has different designations in the literature depending on the communities and the time period. In this paper, "views" and "modes" are considered as synonyms referring to the same concept.

P. Flach et al. (Eds.): ECML PKDD 2012, Part I, LNCS 7523, pp. 130-142, 2012.

(C) Springer-Verlag Berlin Heidelberg 2012 
global view which is made from the union of all mono-views while the latter proposes to combine the decisions of different single-view based classifiers. On the one hand, the principle of the $E F$ strategy is to directly learn the combination of all the features that minimizes a loss function. The drawback of this approach is to combine artificially different data sources (with possibly different semantics), hence increasing the dimension of the feature space, which could result in a higher variance of the generalization error. On the other hand, the goal of $L F$ strategy is to obtain a consensus among independent specialized classifiers leading to a lower generalization error. The drawback of this approach lies in its inability to detect interactions; in other words, it miss the opportunity to capture correlations between different views which may also lead to a lower generalization error and a better understanding of the underlying data.

In this work, we propose a novel multi-modal $(M M)$ framework that tries to exploit the best of the two strategies, while avoiding their drawbacks. It offers the $E F$ 's advantage of capturing interactions across the different modes while, in the meantime, owning the $L F$ 's advantage of learning a weighted combination of the input scores in order to, first, detect a high consensus among the hypotheses and, second, have a better understanding of interactions between views. To achieve this goal, we propose to extend the standard "Rocchio" classification algorithm (see [4]) to the multi-modal case by computing "cross-modal" scores that measure the interaction between pairs of modes. In a nutshell, the "Rocchio" classification algorithm builds prototypes (centroids) for each class and classifies a new instance by linearly combining the similarity of this instance with the class prototypes. In the same vein, we propose in this work to extend the notion of (mono-modal) class prototype by defining for each class: (1) one (mono-modal) centroid per mode (which corresponds to the prototypes defined in the standard "Rocchio" classification algorithm) and (2) two "cross-modal" centroids per pair of modes. While simple centroids aim at focusing on the mono-modal aspects of the data, "cross-modal" centroids bias one mode by using the other one and hence take into account the multi-modal aspects of the data. When classifying a new input, it is compared with these different centroids (mono- and cross-modal) using a similarity function (for instance, the cosine or any similarity measure of information retrieval). These similarity scores form then the inputs of a linearly weighted $L F$ process.

This novel $M M$ framework is benchmarked on a social media corpus - namely the ENRON corporate email data set - on two different tasks: a foldering task and a recipient proposal task. For the foldering task, we consider the seven mailboxes selected by [5]. These mailboxes have been intensively benchmarked due to their public availability. Our framework is compared to $E F$ and $L F$ which constitute the state-of-the art for these tasks on this data set (see for instance the recent work of [6]). For the recipient proposal task, we evaluate the proposed framework on three mailboxes among the ones having the largest amount of messages.

We show that, thanks to the introduced cross-modal scores, our $M M$ framework outperforms the state-of-the-art on this public data set for both tasks. 
To summarize, the main contributions of this paper are the following:

- It introduces a new competitive framework which can be used for classification in case of multi-modal inputs.

- It proposes to compute cross-modal centroids which reflect the multi-modal aspects of the data.

- It shows on various mailboxes of the ENRON data set that the proposed method outperforms established methods, i.e. the $E F$ and the $L F$, on foldering and recipient proposal tasks.

\section{Problem Statement}

More concretely, the multi-modal classification problem may be formalized as follows: We have at our disposal a collection of $N_{d}$ data instances represented by $M$ different data matrices $\mathbf{X}^{(m)}$ of size $N_{d} \times N_{f}^{(m)}$, where $N_{f}^{(m)}$ is the number of features associated with the mode $m \in \mathcal{M}$ (the set of modes $\{1, \ldots, M\}$ ). The vector $\mathbf{x}_{d \bullet}^{(m)}$ denotes the row $d$ of $\mathbf{X}^{(m)}$ and $x_{i, j}^{(m)}$ denotes the entry $(i, j)$ of the same matrix. $\mathbf{X}$ is the matrix obtained by concatenating the different $\mathbf{X}^{(m)}$ matrices in an ascending mode order, i.e. $\mathbf{X}=\left[\mathbf{X}^{(1)} \mathbf{X}^{(2)} \ldots \mathbf{X}^{(|M|)}\right]$. The vector $\mathbf{x}_{d \bullet}^{(\bullet)}$ denotes the row $d$ of this matrix.

For learning, we have a set of labeled multi-modal data instances $\mathcal{L}=$ $\left\{\mathbf{x}_{d \bullet}^{(\bullet)}, y_{d}\right\}, d \in\left[1, N_{d}\right], y_{d} \in \mathcal{C}, \mathbf{x}_{d \bullet}^{(\bullet)} \in \mathcal{X} . \mathcal{C}$ is the set of the different labels: $\left\{c_{1}, \ldots, c_{|\mathcal{C}|}\right\}$ and $\mathcal{X}$ the set of multi-modal data instances.

We want to design a model $M_{c} \in \mathcal{M}$ (the set of multi-modal models) for each class $\mathrm{c} \in \mathcal{C}$ and a multi-modal classification function $F: \mathcal{X} \times \mathcal{M} \rightarrow$ $\mathbb{R}$. The predicted class of an unlabeled multi-modal point $\mathbf{x}_{u}^{(\bullet)}$ will be $\widehat{y}=$ $\operatorname{argmax}_{c} F\left(\mathbf{x}_{u \bullet}^{(\bullet)}, M_{c}\right)$.

One way to solve the problem consists of learning the classification models $M_{c}$ 's directly in the feature space which consists of the union of all modes. In this case, we are using an early fusion $(E F)$ approach. However, as suggested by [7], in order to achieve good performance, each mode should be normalized separately before combination. This is mostly to make features comparable with respect to the range of values across the different modes and features.

Another standard approach consists of learning separately the different classification models $M_{c}^{m}$ 's for each mode $m$. After learning, the decision function consists of a linear combination of the different scores obtained on each mode $m$.

$$
\widehat{y}=\operatorname{argmax}_{c} \sum_{m} \alpha_{c}^{m} F\left(\mathbf{x}_{u \bullet}^{(m)}, M_{c}^{m}\right)
$$

The weights $\alpha_{c}^{m}$ attributed to the different modes for each class are generally tuned on a independent validation set. This method is called late fusion $(L F)$ as opposed to early fusion. Note that most of the proposed and widely used learning to rank systems are based on the linear combination of features [8, 9].

The advantage of the early fusion lies in its ability to capture relations between features across different modes. Furthermore, it avoids the supplementary 
task of tuning hyper-parameters needed in the case of a late fusion approach. However, the early fusion suffers from combining artificially into one vector space multiple sources having different semantics. Furthermore, the level of sparsity may change across modes. Indeed, for instance, image features are dense, while bag-of-words document vectors are sparse and social-media participant vectors are binary sparse vectors. Another drawback of the early fusion method is to increase drastically the dimension of the feature space which results in increasing the variance of the generalization error. Hence, the late fusion strategy which consists of combining different expert models results generally in a lower variance. Its drawback is its inability to capture any possible combination of features across different modalities that may result in a better prediction.

In the remainder, we introduce a new framework which takes a decision based on a linear combination of multiple experts and therefore is $L F$-based. However, while the standard $L F$ does not take into account the interactions across the multiple modes, this framework proposes to exploit the multi-modal aspects of the data. The proposed classification procedure, inspired by the trans-media relevance feedback in information retrieval [10], consists of the following steps: (1) off-line modeling of each class with a representative centroid per mode $m$ obtained after aggregating all the mode $m$ portion of the data instances, (2) offline modeling of each class with two representative centroids per pair of modes (i.e. $(m)\left[m^{\prime}\right]$ and $\left.\left(m^{\prime}\right)[m]\right)$ obtained after aggregating the mode $m$ portion of the nearest neighbors computed through the mode $m^{\prime}$ portion of the data instances, (3) defining similarity scores between unlabeled data points and the different centroids, (4) late (linear) fusion of the obtained scores in order to get a global membership score for each class and (5) applying a simple argmax function on global scores computed for each class in order to predict the class $\widehat{y}$.

\section{Multi-modal Classes Modeling}

Mono-modal Modeling. The off-line training phase consists of learning one model $M_{c}$ for each class $c$. We propose to build a multi-modal centroid vector for each class $c$ which summarizes the subset $\mathcal{L}_{c}$ of all the labeled data instances $\mathcal{L}$ which have class $c$ as label. In other words, $\mathcal{L}_{c}=\left\{\mathbf{x}_{d \bullet \bullet}^{(\bullet)}, c\right\}$, is the set of all data instances that belongs to class $c$. Each centroid is made by aggregating all data instances $d$ of $\mathcal{L}_{c}$.

$$
C^{(m)}(c)=\bigoplus_{d \in \mathcal{L}_{c}} \frac{\mathbf{x}_{d \bullet}^{(m)}}{w_{d}}
$$

$\bigoplus$ is an aggregation operator which may be an average, a max, a min. $w_{d}$ is a weighting factor which counts the number of classes the document $d$ belongs to. Concretely, the intuition is that in a multi-label setting, as for instance in the recipient proposal task (see Section 5.2), a multi-labeled document is less representative of the classes it belongs to than a mono-labeled document considered as more specific to the class. 
Cross-modal Modeling. So far, the centroids are mono-modal and can only capture the modality in which they are defined. Therefore, following our main goal to capture interactions across modes, we define "cross-centroids". A cross-centroid in mode $m$ is made by aggregating the $m$-mode portion of different nearest neighbors observations $\mathbf{x}_{d^{\prime} \bullet}^{(m)}$. The nearest-neighbors are chosen in $\mathcal{L}$ according to a specific mode $m^{\prime}$ resulting in the cross-centroid $C^{(m)\left[m^{\prime}\right]}(c)$. Notice that, $m$ and $m^{\prime}$ may be equal re-enforcing then the mono-modality aspect of the centroid. More formally, a cross-centroid is computed as follows:

$$
C^{(m)\left[m^{\prime}\right]}(c)=\bigoplus_{d^{\prime} \in \mathrm{NN}\left(\mathbf{x}_{d \bullet}^{\left(m^{\prime}\right)}\right), d \in \mathcal{L}_{c}} \mathbf{x}_{d^{\prime} \bullet}^{(m)} w\left(\mathbf{x}_{d \bullet}^{\left(m^{\prime}\right)}, \mathbf{x}_{d^{\prime} \bullet}^{\left(m^{\prime}\right)}\right)
$$

where $N N\left(x^{\left(m^{\prime}\right)}\right)$ is the "nearest neighbors" function returning the set of nearest neighbors of $x$ in the mode $m^{\prime}$ and using any traditional similarity measure of information retrieval, the function $w\left(\mathbf{x}_{d \bullet}^{\left(m^{\prime}\right)}, \mathbf{x}_{d^{\prime} \bullet}^{\left(m^{\prime}\right)}\right)$ gives a weight proportional to the (mono-modal) similarity between both elements.

\section{Multi-modal Late Fusion}

To assess the affinity of an unlabeled observation to a specific class $c$, we compute its global similarity with the different centroids (i.e. mono modal and crossmodal) representing each class. The multi-modal unlabeled input, $\mathbf{x}_{u \bullet}^{(\bullet)}$, is compared mode by mode with the different centroids. Then, a global membership score is deduced from a linear combination of each mode contribution. The global score is thus computed as follows:

$$
\begin{aligned}
R S V\left(c, \mathbf{x}_{u \bullet}^{(\bullet)}\right)= & \sum_{m} \alpha_{c, m} \operatorname{sim}\left(C^{(m)}, \mathbf{x}_{u \bullet}^{(m)}\right) \\
& +\sum_{m} \sum_{m^{\prime}} \beta_{c, m, m^{\prime}} \operatorname{sim}\left(C^{(m)\left[m^{\prime}\right]}, \mathbf{x}_{u \bullet}^{(m)}\right)
\end{aligned}
$$

where $\alpha_{c, m}$ and $\beta_{c, m, m^{\prime}}$ are positive weights summing up to 1 . The "sim" function may be any traditional similarity measure used in information retrieval. The first part of Equation 4 denotes the simple sum of the similarities of each mode. This part does not cover any interaction across the modes. The last part of the equation aims at capturing the interactions across the different modes by computing the similarity with cross-modal centroids. In the remainder, we will show empirically that computing these cross-modal similarities lead to better performance for multi-modal categorization tasks. As with late fusion, the hyperparameters can be learned in order to maximize a specific utility function (by cross-validation), for example: precision@1 or NDCG@10. 


\section{$5 \quad$ Experiments and Discussions}

\subsection{The ENRON Data Set}

The introduced multi-modal $(M M)$ framework is validated on the ENRON dataset. This dataset consists of a set of vectors and matrices that represent the whole ENRON corpus [see, e.g., 11], after linguistic preprocessing and metadata extraction. The linguistic preprocessing consists of removing some particular artefacts of the collection (for instance some recurrent footers, that have nothing to do with the original collection but indicate how the data were extracted), removing headers for emails (From/To/... fields), removing numerals and strings formed with non-alphanumeric characters, lowercasing all characters, removing stopwords as well as words occurring only once in the collection. There are two types of documents: documents are either (parent) emails or attachments (an attachment could be a spreadsheet, a power-point presentation, ...; the conversion to standard plain text is already given by the data provider). The ENRON collection contains 685,592 documents (455,449 are parent emails, 230,143 are attachments) extracted from 151 different mailboxes. We decided to process the attachments simply by merging their textual content with the content of the parent email, so that we have to deal only with parent emails. For parent emails, we have not only the content information, but also metadata. The metadata consist of:

- the custodian (i.e. the person who owns the mailbox from which this email is extracted);

- the location (i.e. the folder decomposition of each mailbox owner);

- the date or timestamp;

- the Subject field (preprocessed in the same way as standard content text);

- the From field;

- the To field ;

- the CC field.

After preprocessing, we summarize the data by two views for each data point: the textual view and the social view. The textual view consists of a bag-of-word representation of the aggregation of the "Body" vector, the "Attachment" vector and "Subject" vector. The social view is a vector in the bag-of-participants space which is the aggregation of the "From" vector, the "To" vector and the "Cc" vector.

For the foldering task, among the 151 available mailboxes, we consider 7 mailboxes having a sufficient amount of folders selected in [5]. Furthermore, we removed folders which are not specific to the considered mailbox but which have been automatically generated by an email client software. For instance, the folders "All documents", "Calendar", "Sent mail", "Deleted Items", "Inbox", "Sent Items", "Unread Mail", "Contacts" and "Drafts" have been discarded from the evaluation. Statistics on the seven selected folders are reported in Table 1. For the recipient proposal task, we report results for three mailboxes among the five having the largest amount of emails in the collection, namely: Vince J. Kaminski, Jeff Dasovich and Tana Jone's mailboxes. 
Table 1. Folders distribution among of the preprocessed mailboxes used for the foldering task

\begin{tabular}{cccccc}
\hline \hline \multicolumn{2}{c}{ Mailbox } & \#Folders Total \#em Min & \#em. Max \#em. Aver \# em/Folder \\
\hline Beck & 60 & 258 & 1 & 27 & 4.30 \\
Farmer & 27 & 1689 & 1 & 528 & 62.56 \\
Kaminski & 32 & 842 & 1 & 120 & 26.31 \\
Kitchen & 51 & 4162 & 1 & 784 & 81.61 \\
Lokay & 14 & 1837 & 1 & 915 & 131.21 \\
Sanders & 20 & 411 & 2 & 181 & 20.55 \\
Williams & 21 & 2043 & 1 & 1076 & 97.29 \\
\hline
\end{tabular}

\subsection{Benchmark Protocol}

The proposed model is tested on two different mail management tasks: (1) email foldering whose goal is to retrieve the correct folder in which an email should go using all its information (i.e. textual content and social metadata), (2) recipient proposal whose goal is to automatically propose recipient candidates for a new written message based only on the textual part of the email. It is important to note that the temporal aspect plays here a big role. Indeed, it would be generally easier to predict the recipient of new emails based on recent posts than on old ones. This comes from the fact that generally emails are part of a global discussion thread. In case of foldering, users may create folders, delete or move emails after an amount of time, for instance to the "Trash" folder to free memory on the server. We could introduce the temporal aspect in our model when building centroids by weighting the contribution of each message using the timestamp meta data. However, in order to compare more fairly with stateof-the-art $L R$ and $E R$ fusion methods we decide to not include the temporal information directly into the model. Instead, we decide to make training-test splits which reflect the sequential aspect of the data such that the compared methods are tested on more recent emails than those used during the training phase. Hence, we assume that the emails of the collection are sorted by increasing order of their timestamp.

For the foldering task, we consider training sets composed by $50 \%$ of the mailbox. After learning, the goal is to predict the folder of the next $10 \%$ emails in the temporal sequence. For example, when considering a training set made from the first $50 \%$ of the collection (i.e. in terms of timestamp), the test set then consists of the emails in the interval 50\%-60\%. By time-shifting the training and the test sets by $10 \%$, we may consider training on the emails going from $10 \%$ to $60 \%$ and test on the next $10 \%$, and so on. So that, finally, we define 5 possible training and test sets. The different methods require some hyper-parameters to be set. For this purpose, during each training phase, hyper-parameters are tuned internally by dividing the $50 \%$ training set into $40 \%$ internal-training and $10 \%$ internal-test. For the recipient proposal task, we consider training sets and test sets made each by $10 \%$ of the mailbox. By time-shifting by $10 \%$ the training-test sets we define 9 different splits. The first two splits are used as validation set for 
Table 2. Table presenting the results for a foldering task averaged on 5 chronological ordered training-test pairs for the Beck's, Farmer's, Kaminski's and Kitchen's mailboxes. The state-of-the art methods late fusion $(L F)$ and early fusion $(E F)$ are compared with the proposed multi-modal framework. The reported performance measure for this mono-label classification task are the Recall@1,@5, @10, the NDCG@10 and the NDCG. The hyper-parameters of each algorithm has been tuned on an independent validation set.

\begin{tabular}{ccccccc}
\hline \hline Alg. & R@1 & R@3 & R@5 & R@10 & NDCG@10 & NDCG \\
\hline \multicolumn{7}{c}{ Beck's mailbox } \\
\hline MM & $\mathbf{0 . 5 5}+/-0.15$ & $\mathbf{0 . 6 8}+/-0.12$ & $\mathbf{0 . 7 1}+/-0.08$ & $\mathbf{0 . 7 7}+/-0.11$ & $\mathbf{0 . 6 6}+/-0.11$ & $\mathbf{0 . 6 8}+/-0.10$ \\
LF & $0.42+/-0.1$ & $0.57+/-0.06$ & $0.62+/-0.06$ & $0.70+/-0.04$ & $0.56+/-0.06$ & $0.60+/-0.06$ \\
EF & $0.46+/-0.14$ & $0.63+/-0.15$ & $0.65+/-0.11$ & $0.69+/-0.09$ & $0.59+/-0.12$ & $0.63+/-0.10$ \\
\hline \multicolumn{7}{c}{ Farmer's mailbox } \\
\hline MM & $0.68+/-0.11$ & $\mathbf{0 . 8 2}+/-0.09$ & $\mathbf{0 . 8 7}+/-0.08$ & $\mathbf{0 . 8 9}+/-0.07$ & $\mathbf{0 . 7 9}+/-0.08$ & $\mathbf{0 . 8 1}+/-0.08$ \\
LF & $0.65+/-0.14$ & $0.79+/-0.13$ & $0.81+/-0.12$ & $0.86+/-0.09$ & $0.76+/-0.12$ & $0.78+/-0.11$ \\
EF & $0.68+/-0.13$ & $0.77+/-0.12$ & $0.81+/-0.11$ & $0 / 85+/-0.08$ & $0.76+/-0.11$ & $0.78+/-0.10$ \\
\hline \multicolumn{7}{c}{ Kaminski's mailbox } \\
\hline MM & $\mathbf{0 . 5 4}+/-0.20$ & $\mathbf{0 . 6 8}+/-0.21$ & $\mathbf{0 . 7 3}+/-0.16$ & $\mathbf{0 . 8 2}+/-0.18$ & $\mathbf{0 . 6 7}+/-0.19$ & $\mathbf{0 . 7 0}+/-0.18$ \\
LF & $0.44+/-0.19$ & $0.61+/-0.25$ & $0.67+/-0.22$ & $0.77+/-0.17$ & $0 / 60+/-0.19$ & $0.63+/-0.19$ \\
EF & $0.51+/-0.22$ & $0.61+/-0.21$ & $0.67+/-0.23$ & $0.78+/-0.21$ & $0.63+/-0.21$ & $0.66+/-0.20$ \\
\hline \multicolumn{7}{c}{ Kitchen's mailbox } \\
\hline MM & $0.42+/-0.13$ & $0.65+/-0.07$ & $0.77+/-0.07$ & $0.87+/-0.08$ & $0.63+/-0.09$ & $0.65+/-0.08$ \\
LF & $0.41+/-0.12$ & $0.65+/-0.16$ & $0.75+/-0.13$ & $0.81+/-0.14$ & $0.61+/-0.13$ & $0.64+/-0.12$ \\
EF & $0.44+/-0.12$ & $0.64+/-0.12$ & $0.74+/-0.10$ & $0.83+/-0.10$ & $0.63+/-0.11$ & $0.66+/-0 / 09$ \\
\hline \hline
\end{tabular}

tuning the hyper-parameters of the different methods. The remaining splits are used for assessing the performance of the different algorithms.

\subsection{Classification Models and Tuning}

The benchmarked classification models are (1) the $M M$ model proposed in this paper, the (2) EF model and the (3) $L F$ model. For the $E F$ and $L F$ fusion models we use a one-vs-rest logistic regression classifier with a 12-norm regularization. This provides, after normalization, the posterior probability of each class given a test data point. As previously said, the regularization parameter is internally tuned during training for the foldering task, or tuned on an independent validation set for the recipient proposal task. For the $L F$ strategy, the best convex combination (i.e. achieving the best performance in terms of NDCG@10 on a independent data set) of the mono-modal classifiers is kept for the test.

For the $M M$ model, a set of parameters has to be learned for each class corresponding to the weights given to each centroid by the model. The class parameters are learned using a logistic regression where a positive target $(+1)$ is associated to data points that belongs to the class and negative target $(0)$ for unlabeled points. For classes with less than 30 data points, we use an uniform convex combination of the weights.

Note that the number of nearest-neighbors used for computing "cross-modal" centroids has been set to 10 . The results obtained on 5, 20 and 30 nearestneighbors lead to the same observations. 
Table 3. Table presenting the results for a foldering task averaged on 5 chronological ordered training-test pairs for the Lokay's, Sanders's and Williams's mailboxes. The state-of-the art methods late fusion $(L F)$ and early fusion $(E F)$ are compared with the proposed multi-modal framework. The reported performance measure for this monolabel classification task are the Recall@1,@5, @10, the NDCG@10 and the NDCG. The hyper-parameters of each algorithm has been tuned on an independent validation set.

\begin{tabular}{ccccccc}
\hline \hline Alg. & R@1 & R@3 & R@5 & R@10 & NDCG@10 & NDCG \\
\hline \multicolumn{7}{c}{ Lokay's mailbox } \\
\hline MM & $0.80+/-0.04$ & $0.92+/-0.05$ & $0.95+/-0.04$ & $0.96+/-0.04$ & $0.89+/-0.04$ & $0.89+/-0.04$ \\
LF & $0.77+/-0.04$ & $0.90+/-0.05$ & $0.94+/-0.04$ & $0.96+/-0.04$ & $0.87+/-0.04$ & $0.87+/-0.04$ \\
EF & $0.81+/-0.05$ & $0.91+/-0.06$ & $0.95+/-0.05$ & $0.96+/-0.04$ & $0.89+/-0.05$ & $0.89+/-0.05$ \\
\hline \multicolumn{7}{c}{ Sanders's mailbox } \\
\hline MM & $\mathbf{0 . 8 2}+/-0.12$ & $\mathbf{0 . 8 6}+/-0.13$ & $\mathbf{0 . 8 8}+/-0.10$ & $\mathbf{0 . 9 2}+/-0.12$ & $\mathbf{0 . 8 6}+/-0.12$ & $\mathbf{0 . 8 7}+/-0.11$ \\
LF & $0.70+/-0.13$ & $0.84+/-0.13$ & $0.86+/-0.13$ & $0.90+/-0.15$ & $0.80+/-0.13$ & $0.82+/-0.12$ \\
EF & $0.78+/-0.14$ & $0.82+/-0.14$ & $0.86+/-0.16$ & $0.89+/-0.15$ & $0.83+/-0.14$ & $0.84+/-0.13$ \\
\hline \multicolumn{7}{c}{ Williams's mailbox } \\
\hline MM & $0.68+/-0.34$ & $0.80+/-0.38$ & $0.81+/-0.38$ & $0.81+/-0.36$ & $0.76+/-0.36$ & $0.77+/-0.33$ \\
LF & $0.74+/-0.37$ & $0.80+/-0.39$ & $0.81+/-0.38$ & $0.81+/-0.38$ & $0.78+/-0.38$ & $0.79+/-0.35$ \\
EF & $0.74+/-0.37$ & $0.80+/-0.38$ & $0.81+/-0.38$ & $0.78+/-0.37$ & $0.78+/-0.37$ & $0.79+/-0.34$ \\
\hline \hline
\end{tabular}

\subsection{Results and Discussion}

Foldering Task: The obtained results for the $M M, E F$ and $L F$ are reported in Table 2 and Table 3 . The retrieval measure performance are the recall at rank 1 (R@1), the recall at rank $3(\mathrm{R} @ 3)$, the recall at rank $5(\mathrm{R} @ 5)$ and the recall at rank $10(\mathrm{R} @ 10)$, knowing that, for each data point, there is only one relevant folder. We measure also the normalized discounted cumulative gain, limited to rank 10 (NDCG@10) and on the whole set of folders (NDCG). The reported scores are the results averaged over the 5 sequential training-test pairs. Clearly, for 4 of the 7 mailboxes (namely: Beck, Farmer, Kaminski and Sanders) the proposed $M M$ framework outperforms the $E F$ and the $L F$ strategies. The scores in bold mean that the performances are significantly better (verified by a signed test with a p-value $<0.05)$. Moreover, the $E F$ and $L F$ methods never outperforms the $M M$ approach on all the measures simultaneously. Although the $L F$ is generally the preferred approach for a foldering task on this data set (see, e.g. [6]), we observe in our tests that $E F$ is always better or equivalent for all the performance measures than $L F$. Hence, we argue that often $E F$ is not correctly used. Indeed, it is important to normalize independently each view before fusion due to the semantic difference that may exist between the views. The results reported on William's mailbox have a large variance. This is because, at the second time step, new folders have been created by the user for which no emails or a few were present in the training. In the real world, this realistic case often happen, therefore designing sequential training-test splits is critical in order to fairly assess the performance of the system.

Recipient Proposal. The results are reported for three different mailboxes in Table 4. The recipient proposal task is a multi-label classification task for which the performance are measured using the macroF1 (maF1), the mean average precision (MAP) and the NDCG. For the MM framework, we also report the 
Table 4. Averaged performance measures on 7 different time slots of size $10 \%$ (i.e. training size of $10 \%$ ) for Kaminski's, Dasovich's and Jone's mailboxes on a recipient proposal task. The state-of-the art methods late fusion $(L F)$ and early fusion $(E F)$ are compared with the proposed multi-modal framework. The reported performance measure for this multi-label classification task are the maF1, the MAP and the NDCG. The hyper-parameters of each algorithm has been tuned on an independent validation set.

\begin{tabular}{|c|c|c|c|}
\hline Model & maF1 & MAP & NDCG \\
\hline \multicolumn{4}{|c|}{ Kaminski's mailbox } \\
\hline$C^{\text {(text) }}$ & $0.16+/-0.03$ & $0.24+/-0.05$ & $0.40+/-0.07$ \\
\hline$C^{\text {(text) }[\text { text }]}$ & $0.38+/-0.06$ & $0.44+/-0.09$ & $0.55+/-0.11$ \\
\hline$C^{\text {(text)[participant] }}$ & $0.11+/-0.02$ & $0.13+/-0.03$ & $0.24+/-0.04$ \\
\hline$C^{\text {(participant) }}$ & $0.04+/-0.01$ & $0.11+/-0.01$ & $0.26+/-0.04$ \\
\hline$C^{\text {(participant)[participant] }}$ & $0.11+/-0.01$ & $0.21+/-0.02$ & $0.37+/-0.04$ \\
\hline$C^{\text {(participant) }[\text { text }]}$ & $0.02+/-0.01$ & $0.04+/-0.01$ & $0.15+/-0.02$ \\
\hline$\alpha C^{\text {(text) }}+(1-\alpha) C^{\text {(participant })}$ & $0.18+/-0.03$ & $0.26+/-0.05$ & $0.42+/-0.07$ \\
\hline $\mathrm{EF}$ & $0.29+/-0.16$ & $0.34+/-0.18$ & $0.45+/-0.19$ \\
\hline $\mathrm{LF}$ & $0.30+/-0.16$ & $0.35+/-0.18$ & $0.46+/-0.19$ \\
\hline$R S V$ & $\mathbf{0 . 4 0}+/-0.05$ & $\mathbf{0 . 4 6}+/-0.08$ & $0.57+/-0.11$ \\
\hline \multicolumn{4}{|c|}{ Dasovich's mailbox } \\
\hline & $0.61+/-0.14$ & $0.25+/-0.06$ & $0.46+/-0.04$ \\
\hline$C^{\text {(text) }[\text { text }]}$ & $0.66+/-0.14$ & $0.28+/-0.06$ & $0.48+/-0.07$ \\
\hline$C^{(\text {text })[\text { participant }]}$ & $0.57+/-0.14$ & $0.25+/-0.03$ & $0.47+/-0.02$ \\
\hline$C^{\text {(participant) }}$ & $0.62+/-0.08$ & $0.30+/-0.04$ & $0.49+/-0.03$ \\
\hline$C^{(\text {participant)[participant] }}$ & $0.74+/-0.15$ & $0.38+/-0.04$ & $0.54+/-0.04$ \\
\hline$C^{(\text {participant })[\text { text] }}$ & $0.78+/-0.09$ & $0.37+/-0.04$ & $0.49+/-0.03$ \\
\hline$\alpha C^{(\text {text })}+(1-\alpha) C^{(\text {participant })}$ & $0.67+/-0.12$ & $0.31+/-0.04$ & $0.50+/-0.04$ \\
\hline $\mathrm{EF}$ & $36+/-0$ & $0.41+/-0.06$ & $0.52+/-0.06$ \\
\hline $\mathrm{LF}$ & $7+/-$ & $1+/-$ & $0.52+1$ \\
\hline$R S V$ & $\mathbf{0 . 7 9}+/-0.07$ & $0.40+/-$ & $0.55+/$ \\
\hline \multicolumn{4}{|c|}{ Jone's mailbox } \\
\hline$C^{(\text {tex }}$ & $0.86+/-0.06$ & $0.41+/-0.07$ & $0.55+/-0.05$ \\
\hline$C^{\text {(text) }[\text { text }]}$ & $0.90+/-0.05$ & $0.45+/-0.06$ & $0.56+/-0.05$ \\
\hline$C^{(\text {text })[\text { participant }]}$ & $0.83+/-0.07$ & $0.39+/-0.06$ & $0.54+/-0.04$ \\
\hline$C^{\text {(participant) }}$ & $0.80+/-0.05$ & $0.40+/-0.07$ & $0.55+/-0.05$ \\
\hline$C^{\text {(participant)[participant] }}$ & $0.86+/-0.07$ & $0.47+/-0.06$ & $0.60+/-0.04$ \\
\hline$C^{\text {(participant)[text] }}$ & $0.79+/-0.04$ & $0.38+/-0.06$ & $0.54+/-0.05$ \\
\hline$\alpha C^{(\text {text })}+(1-\alpha) C^{(\text {participant })}$ & $0.86+/-0.04$ & $0.41+/-0.06$ & $0.55+/-0.04$ \\
\hline $\mathrm{EF}$ & $0.46+/-0.12$ & $0.50+/-0.09$ & $0.59+/-0.06$ \\
\hline $\mathrm{LF}$ & $0.46+/-0.12$ & $0.12+/-0.09$ & $0.59+/-0.06$ \\
\hline$R S V$ & $\mathbf{0 . 9 1 + / - 0 . 0 4}$ & $\mathbf{0 . 5 0}+/-0.06$ & $\mathbf{0 . 6 0}+/-0.05$ \\
\hline
\end{tabular}


results obtained individually by each centroid. Moreover, in addition of reporting the results obtained by the $E F$ and $L F$ baselines, we also report the results obtained by the MM-baseline which consists of using only a combination of the mono-modal centroids. (in other words, the combination of the textual centroid and the social centroid : $\left.\alpha C^{\text {(text) }}+(1-\alpha) C^{\text {(participant) }}\right)$. On the three tested mailboxes, the proposed MM framework outperforms the $E F$ and the $L F$ and the simple combination of the textual and the social centroids. The scores in bold mean that the performances are significantly better (verified by a signed test with a p-value $<0.05)$. Notice that, on the Kaminski's mailbox, textual centroids obtained a better score than the social centroids, while on the Dasovitch's mailbox social centroids obtained a better score than the textual centroids.

\section{Related Work}

In a fully supervised setting, as pointed out in [3], multi-view learning usually performs worse than learning on the union of all views. Hence, a few has been done in this field. For instance, [12] proposed a method that combines a two stage learning (KCCA followed by SVM) into a single optimization termed "SVM$2 \mathrm{~K}$ ". Others have considered working on a graph representation of the data. For instance, [13] exploited hyperlinks between web pages in order to improve traditional classification tasks using only the content. [14] studied the composition of kernels in order to improve the performance of a soft-margin support vector machine classifier. 15, 16] used both local text in a document as well as the distribution of the estimated classes of other documents in its neighborhood, to refine the class distribution of the document being classified. Their framework has been tested for the semi-supervised and fully-supervised classification as well. More recently, 17] proposes to learn the weights of a namely "supervised random walk" using both the information from the network structure and the attribute data.

In this paper we introduce an extension of the classical Rocchio classification algorithm also known as the nearest centroid or nearest prototype classifier (see [18]) to the multi-modal case. An extension of this algorithm using kernel-based similarities has been introduced in [19]. A probabilistic variant of the Rocchio classifier has been proposed in [20].

\section{Conclusions}

In this work, we introduced a novel and simple algorithm in order to deal with multi-modal fully supervised classification. To this purpose, we extended the traditional Rocchio classification algorithm by defining mono-modal and multimodal centroids. The introduced framework has the advantage of searching for a high consensus among views using scores reflecting the interactions between the different existing modes. We showed on two different tasks - a foldering task and recipient prediction task - that the proposed multi-modal framework outperforms state-of-the-art approaches: the early fusion and the late fusion. As 
further research, we would like to investigate multi-view learning with latent spaces and interactions between latent variables.

\section{References}

[1] Abney, S.P.: Bootstrapping. In: Proceedings of the 40th Annual Meeting of the Association for Computational Linguistics, pp. 360-367 (2002)

[2] Zhu, X.: Semi-supervised learning literature survey. Technical report (2008)

[3] Ruping, S., Scheffer, T.: Learning with multiple views proposal for an icml workshop. In: Proceedings of the ICML 2005 Workshop on Learning With Multiple Views, Bonn, Germany, August 11, pp. 1-7 (2005)

[4] Manning, C., Raghavan, P., Schutze, H.: Introduction to information retrieval. Cambridge University Press (2008)

[5] Bekkerman, R., McCallum, A., Huang, G.: Automatic categorization of email into folders: Benchmark experiments on enron and sri corpora. Technical report, University of Massachusetts (2004)

[6] Tam, T., Ferreira, A., Lourenço, A.: Automatic Foldering of Email Messages:A Combination Approach. In: Baeza-Yates, R., de Vries, A.P., Zaragoza, H., Cambazoglu, B.B., Murdock, V., Lempel, R., Silvestri, F. (eds.) ECIR 2012. LNCS, vol. 7224, pp. 232-243. Springer, Heidelberg (2012)

[7] Liu, T., Xu, J., Qin, T., Xiong, W., Li, H.: Letor: Benchmark dataset for research on learning to rank for information retrieval. In: Proceedings of SIGIR 2007 Workshop on Learning to Rank for Information Retrieval, pp. 3-10 (2007)

[8] Xu, J., Li, H.: Adarank: a boosting algorithm for information retrieval. In: Proceedings of the 30th Annual International ACM SIGIR Conference on Research and Development in Information Retrieval, pp. 391-398. ACM (2007)

[9] Yue, Y., Finley, T., Radlinski, F., Joachims, T.: A support vector method for optimizing average precision. In: ACM SIGIR Conference on Research and Development in Information Retrieval (SIGIR), pp. 271-278 (2007)

[10] Clinchant, S., Renders, J.-M., Csurka, G.: Trans-Media Pseudo-Relevance Feedback Methods in Multimedia Retrieval. In: Peters, C., Jijkoun, V., Mandl, T., Müller, H., Oard, D.W., Peñas, A., Petras, V., Santos, D. (eds.) CLEF 2007. LNCS, vol. 5152, pp. 569-576. Springer, Heidelberg (2008)

[11] Klimt, B., Yang, Y.: The Enron Corpus: A New Dataset for Email Classification Research. In: Boulicaut, J.-F., Esposito, F., Giannotti, F., Pedreschi, D. (eds.) ECML 2004. LNCS (LNAI), vol. 3201, pp. 217-226. Springer, Heidelberg (2004)

[12] Farquhar, J.D.R., Hardoon, D.R., Meng, H., Shawe-Taylor, J., Szedmák, S.: Two view learning: Svm-2k, theory and practice. In: Proceedings of Advances in Neural Information Processing Systems, pp. 355-362 (2005)

[13] Slattery, S., Mitchell, T.: Discovering test set regularities in relational domains. In: Proceedings of the 7th International Conference on Machine Learning (ICML 2000), pp. 895-902 (2000)

[14] Joachims, T., Cristianini, N., Shawe-Taylor, J.: Composite kernels for hypertext categorisation. In: Proceedings of the International Conference on Machine Learning (ICML 2001), pp. 250-257 (2001)

[15] Chakrabarti, S., Dom, B., Indyk, P.: Enhanced hypertext categorization using hyperlinks. In: Proceedings of the 1998 ACM SIGMOD International Conference on Management of Data, pp. 307-318 (1998) 
[16] Oh, H., Myaeng, S., Lee, M.: A practical hypertext catergorization method using links and incrementally available class information. In: Proceedings of the 23rd International ACM Conference on Research and Development in Information Retrieval (SIGIR 2000), pp. 264-271. ACM (2000)

[17] Backstrom, L., Leskovec, J.: Supervised random walks: predicting and recommending links in social networks. In: Proceedings of the Forth International Conference on Web Search and Web Data Mining, WSDM 2011, Hong Kong, China, pp. 635-644 (2011)

[18] Tibshirani, R., Hastie, T., Narasimhan, B., Chu, G.: Diagnosis of multiple cancer types by shrunken centroids of gene expression. Proceedings of the National Academy of Sciences 99(10) 99(10), 6567 (2002)

[19] Scholkopf, B., Smola, A.: Learning with kernels. The MIT Press (2002)

[20] Joachims, T.: A probabilistic analysis of the rocchio algorithm with tfidf for text categorization. In: Proceedings of International Conference on Machine Learning (ICML 1997), pp. 143-151 (1997) 